\title{
Bronchogenic squamous cell carcinoma metastasizing to Bowman's capsule
}

\author{
LIVIA ROSS \\ From Highland-Alameda County Hospital, Oakland, California, U.S.A.
}

SYNOPSIS A kidney metastasis from a squamous bronchogenic carcinoma is described that showed the unusual feature of occupying Bowman's capsule as well as metastasizing interstitially, producing a neoplastic 'crescent' formation. The lesion is compared with the histological picture of subacute glomerulonephritis. No explanation is offered for this route for metastasis.

The histological appearances of a metastasis to Bowman's capsule could be confused with hyperplastic and hypertrophic parietal epithelium of Bowman's capsule (Allen, 1962), imitating crescent formation, such as is seen in subacute glomerulonephritis. This case illustrates the difference between a primary glomerular disease also involving Bowman's capsule and a metastasis that lines the Bowman's layer producing a cuff of 'hyperplastic' neoplastic cells around an otherwise apparently normal glomerulus.

\section{CASE REPORT}

A 68-year-old white male (D.H.) came to HighlandAlameda County Hospital with the chief complaint of persistent weakness and night sweats. $X$-ray examination showed right upper lobe consolidation. No haemoptysis was present. The patient smoked 20 to 40 cigarettes daily. He had lost $20 \mathrm{lb}$. in the month before admission. On physical examination percussion revealed dullness over the right upper chest area, and bronchoscopy showed tumour protruding from the right upper lobe orifice. A scalene node biopsy was negative. An exploratory thoracotomy showed an unresectable carcinoma of the right lung. After the thoracotomy the patient went downhill and died on his 28th day in hospital. None of the laboratory data revealed pertinent findings.

Necropsy showed a narrow right upper lobe orifice fixed by tumour which had invaded the bronchial wall, extending into the surrounding parenchyma. The hilar nodes were matted, enlarged, and replaced by tumour. The only other metastasis found was in the right kidney, in which a well-circumscribed, grey-white tumour nodule, $2 \times 3 \mathrm{~cm}$., was seen. The cut surface of the nodule appeared dry and granular.

Figure 1 illustrates two glomeruli isolated in a fibrous tissue background in which scattered foci of Received for publication 19 January 1966. metastatic squamous cell carcinoma are seen. In one of the glomeruli two-thirds of Bowman's capsule is replaced by tumour; of the other, approximately

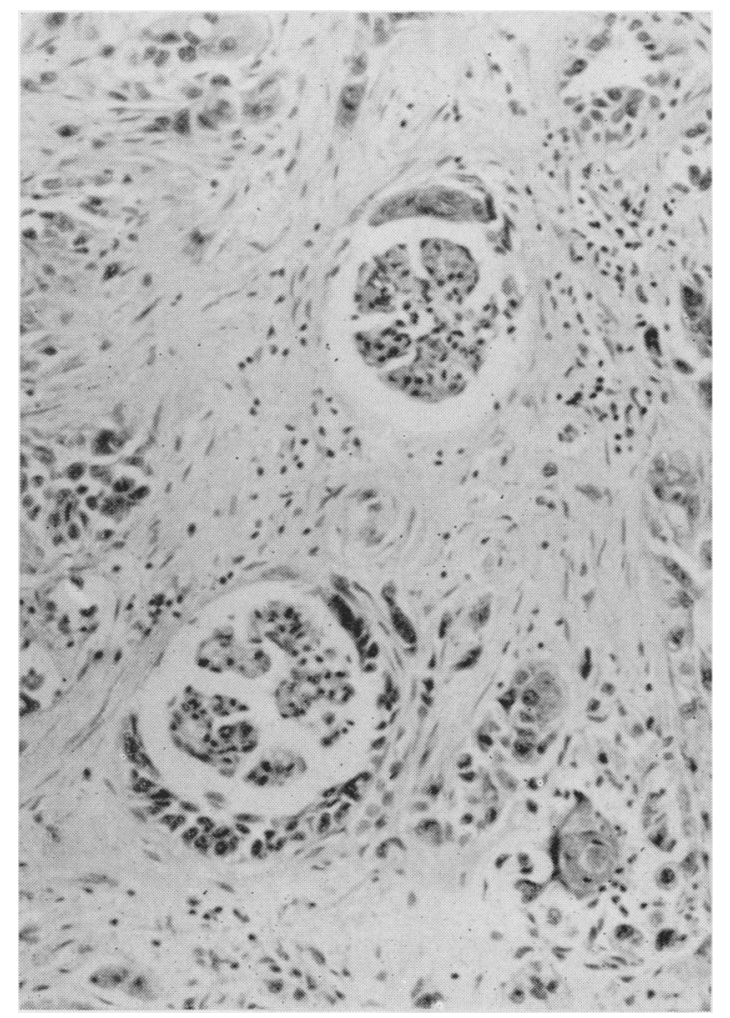

FIG. 1. Two glomeruli surrounded by squamous cell carcinoma metastasis. The Bowman's capsules on their inner aspect show partial tumour replacement. Haematoxylin and eosin. $\times 100$ 


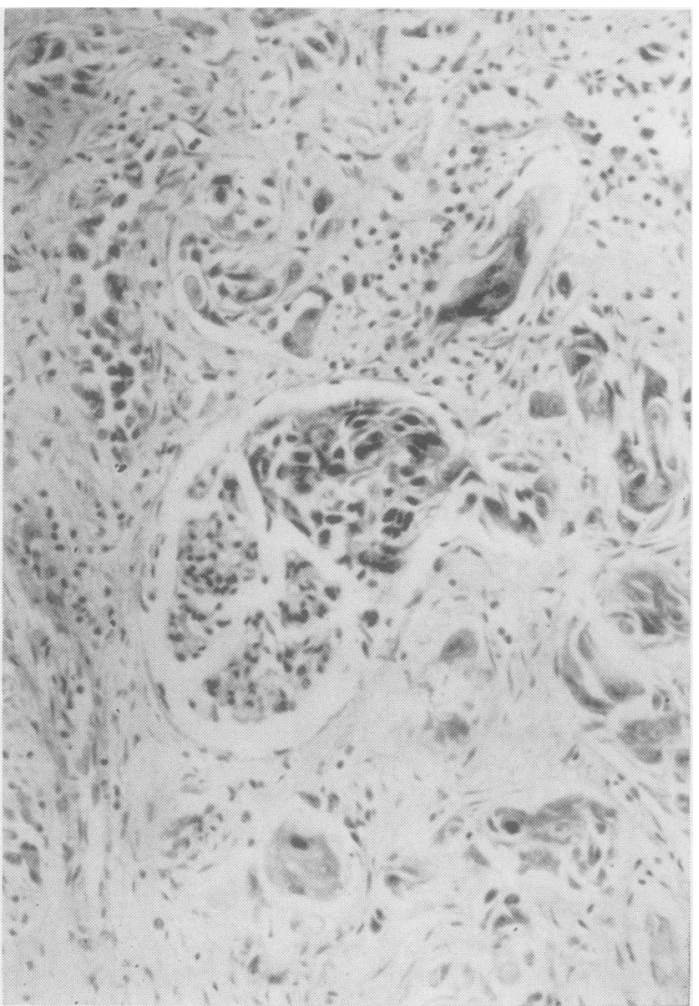

FIG. 2.

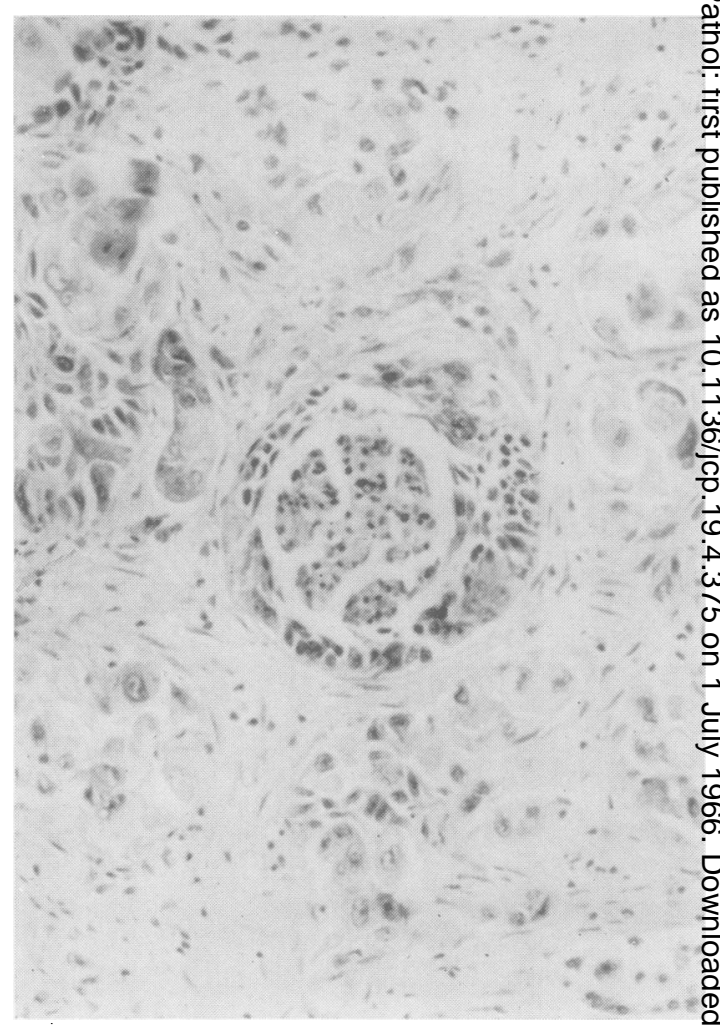

FIG. 3.

FIG. 2. A glomerulus with tumour invagination through the vascular pole. Haematoxylin and eosin. $\times 150$.

FIG. 3. Higher magnification of a glomerulus with distinct basement membrane of the Bowman's capsule lined on the inside by tumour cells. Haematoxylin and eosin. $\times 150$.

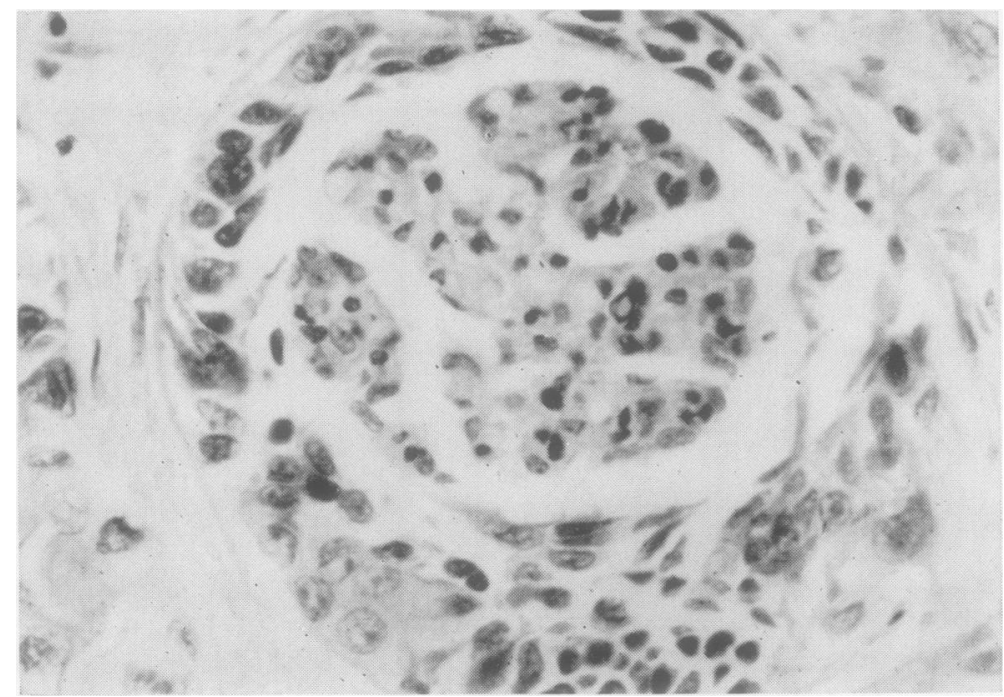

FIG. 4. Highest magnification of glomerulus seen in Fig. 3 to showN the neoplastic cell detail and the glomerular capillary tufts within. Haematoxylin and eosin. $\times 250$. 
one-eighth is lined with carcinoma. The cells show varying amounts of cytoplasm. The nuclei are hyperchromatic and show some nucleolar prominence. Figure 2 shows a glomerulus which has the capillary loops compressed by an invaginating tongue of tumour, which seems to have penetrated through the mesangium of the glomerulus at its base. The displaced glomerular capillary tufts appear to be unaffected by the tumour or by any other disease. Figure 3 illustrates a glomerulus similar to that seen in Fig. 1, emphasizing the site of the metastatic cells, pinpointed even further in Figure 4. This high-power detail of the glomerulus focuses on the neoplastic cells, outlining Bowman's capsule in a multilayered fashion, leaving a healthy, viable-appearing glomerular capillary network in the centre.

\section{DISCUSSION}

I am unable to judge how commonly this type of metastasis occurs because there are so few reports in the literature. In Allen's second edition (1962), a similar case is described but no references or illustrations are given. All that is said is that 'foci of adenocarcinoma may lodge in Bowman's space and may be misinterpreted as hyperplastic and hypertrophic parietal epithelium of Bowman's capsule'.

For a better appreciation of the possible route of this unusual metastasis, it may be helpful briefly to recapitulate the steps in the development of the glomerulus based on Potter's (1965) description. The nephroblastic cells surrounding the ureteral buds form the renal vesicle, which will indent to form an $S$ shape, with the capillaries growing into it to form the glomerulus. At the early stage of development the base of the glomerulus is broad. After further glomerular development the base of the glomerulus begins to be constricted and the space surrounding it becomes sharply separated from that of the tubule, with which it is in communication; a sharp angle is produced in one wall of the terminal part of this tubule and the cells between this angulation and the base of the glomerulus proliferate, flatten, and become part of Bowman's capsule. At the end of this process the glomerular capillaries are covered by an epithelial investment continuous with that comprising Bowman's capsule, which in turn, is continuous with that of the tubule. In the final stage of development, the constriction is so complete that nothing remains of the original broad base of the glomerulus except the afferent and efferent vessels. The outer layer of Bowman's capsule is made up of pavement-like cells.

The glomeruli of the case under discussion show that the 'basement membrane' of Bowman's capsule is preserved. The pavement-like parietal layer represents the replacement by tumour as shown in Figs. 1, 3 , and 4. Figure 2 suggests the portal of entry, namely, the narrow neck of the glomerulus with the tumour, pressing the capillary loops to the periphery. It remains unclear why the tumour cells, after invagination into the glomerulus, encircled the glomerular capillary loops. Could it be that the plasma ultrafiltrate, bathing the parietal layer of Bowman's capsule, was the factor favourable for this mode of spread and growth?

McManus's (1963) description of the crescents is helpful in emphasizing the difference between these structures, as seen in subacute glomerulonephritis, from those described in this case: in the early stage of crescent formation the epithelial cells are being laid down on a fine framework within the space of Bowman's capsule. Later the crescent shows tubular formations, which communicate with the lumen of the proximal tubule. Staining the affected glomeruli with P.A.S. outlines the newly formed tubular basement membranes.

The glomeruli which are the subject of this report showed none of the histological features described as characteristic of primary glomerular disease.

\section{REFERENCES}

Allen, A. C. (1962). The Kidney, 2nd ed. Grune and Stratton, New York.

Potter, E. L. (1965). Arch. Path., 80, 241.

McManus, J. F. A. (1963). In Diseases of The Kidney, edited by M. B. Strauss and L. G. Welt, pp. 255-267. Little, Brown, Boston. 